Relations industrielles

Industrial Relations

\title{
Eric H. NEILSEN : Becoming an O.D. Practitioner. Englewood Cliffs, Prentice Hall, 1984, 280 pp., ISBN 0-13-071555-7
}

\section{Laurent Bélanger}

Volume 39, numéro 4, 1984

URI : https://id.erudit.org/iderudit/050096ar

DOI : https://doi.org/10.7202/050096ar

Aller au sommaire du numéro

Éditeur(s)

Département des relations industrielles de l'Université Laval

ISSN

0034-379X (imprimé)

1703-8138 (numérique)

Découvrir la revue

Citer ce compte rendu

Bélanger, L. (1984). Compte rendu de [Eric H. NEILSEN : Becoming an O.D.

Practitioner. Englewood Cliffs, Prentice Hall, 1984, 280 pp., ISBN 0-13-071555-7].

Relations industrielles / Industrial Relations, 39(4), 809-811.

https://doi.org/10.7202/050096ar

Tous droits réservés @ C Département des relations industrielles de l'Université Laval, 1984
Ce document est protégé par la loi sur le droit d'auteur. L’utilisation des services d'Érudit (y compris la reproduction) est assujettie à sa politique d'utilisation que vous pouvez consulter en ligne.

https://apropos.erudit.org/fr/usagers/politique-dutilisation/ 
aussi question de ses devoirs, notamment celui de préparer sérieusement cette rencontre. Il faut alors transformer ses opinions en arguments.

La question du suivi de l'entretien est posée au chapitre 15 . Au cours des entretiens, les collaborateurs ont parlé devant leur chef mais avec l'espoir que leur parole soit prise en considération, en dehors des limites de son bureau, par référence au besoin à un échelon supérieur. Ils y comptent fermement. Par ailleurs, que faire des traces écrites? L'auteur est clair. Mis à part les désirs de mutations, les demandes de formation... qui doivent être centralisées par le service du personnel, les traces écrites doivent demeurer dans les mains du chef et de son collaborateur seulement. De plus, «le réalisme commande... que toute trace écrite de plus de deux ou trois ans soit détruite et que le collaborateur soit informé de cette procédure». Procéder autrement soulève de sérieux dangers d'indiscrétions et risquerait de miner cette confiance mutuelle si essentielle au succès même de l'entretien.

Finalement, en guise de conclusion, Monsieur X remet les choses au point et découvre de nouvelles dimensions à l'entretien et à sa fonction. Comme le note l'auteur en terminant l'entretien $« . .$. constitue un instrument moins flamboyant que certains autres assurément, moins spectaculaire, mais peut-être plus efficace à court et moyen terme parce qu'il est basé sur des besoins humains et des pratiques sociales indiscutables».

S'il y a certains reproches que l'on peut adresser a cet ouvrage, c'est que l'auteur idéalise à l'occasion les pratiques américaines en matière de gestion de personnel. On peut certes dire qu'en France ce n'est pas l'Amérique. Mais en Amérique on dit, ce n'est pas le Pérou. L'herbe est toujours plus verte dans la cour du voisin. Allez voir!

De plus, les propos de ce livre sont clairement orientés sur des objectifs de formation, de développement, de carrière. Certes, l'auteur mentionne a l'occasion que l'appréciation-évaluation peut aussi servir à déterminer les augmentations de salaires. Cependant, ce sujet n'est pas carrément abordé dans le contexte du traitement qu'il fait de l'entretien.
Finalement, l'auteur néglige de soulever un point qui nous apparaît fondamental. Comment un responsable peut-il être motivé à investir temps et énergie dans l'appréciation formelle de ses subalternes, s'il sait que l'on ne tiendra pas compte de la façon avec laquelle il s'est acquitté de cette responsabilité lorsque, a son tour, il sera évalué par son supérieur immédiat?

Cet ouvrage pratique et intéressant aurait tout intérêt à circuler parmi les gestionnaires de nos organisations. Cela les aiderait à remplir cette responsabilité, si difficile à assumer, qu'est l'entretien d'appréciation.

Roland THÉRIAULT

Ecole des Hautes études commerciales Montréal

Becoming an O.D. Practitioner, par Eric $\mathbf{H}$. Neilsen, Englewood Cliffs, PrenticeHall, 1984, 280 pp., ISBN 0-13-071555-7

À n'en juger uniquement que par le titre, on pourrait croire qu'il s'agit là d'un coffre à outils à l'usage des conseillers en développement des organisations (D.O). D'ailleurs, l'auteur, dans son introduction, réfère à une sorte de livre de cuisine en D.O.; mais il prend là une position bien modeste parce qu'il s'agit beaucoup plus d'un instrument à la portée des conseillers.

Pour ma part, à quelques reprises, dans cette revue même, j'ai présenté le D.O. comme étant beaucoup plus un processus d'intervention dans les organisations qu'un contenu, un processus d'influence exercée par le consultant au sein duquel le consultant devient un modèle de cheminement personnel pour les individus et les groupes qu'il entend aider.

L'auteur de ce volume s'inscrit également dans cette perspective en retenant deux valeurs fondamentales qui sous-tendent tout effort de changement: la candeur (candideness), c'est-a-dire une certaine aptitude chez les individus et les groupes a partager leur expérience ou leur vécu organisationnel; la prise en charge d'une responsabilité per- 
sonnelle pour les gestes qu'ils posent au sein des organisations qui les emploie (selfresponsability).

En reportant sur deux axes le degré auquel ces deux valeurs sont partagés, l'auteur dégage quatre orientations fondamentales au niveau d'un groupe et de la nature des rapports sociaux qui sont vécus par ce groupe de même que quatre types d'organisation.

Les organisations fortement orientées vers le consensus ou la concertation sont composées d'individus qui se perçoivent comme une ressource importante et qui sont fortement impliquées dans la promotion du «bien-être» général de l'organisation. Au niveau des rapports sociaux, l'accent est placé sur le développement de relations collégiales, qui viennent tempérer le caractère formaliste des relations hiérarchiques qu'on connaît habituellement.

Bien entendu, des organisations de ce type présentent un terrain propice pour le développement organisationnel puisqu'elles abritent déjà un fort potentiel de changement. Cependant, ce n'est pas à mon avis le type d'organisation où les conseillers en D.O. seront habituellement appelés à travailler puisqu'une intervention D.O. vise, entre autres objectifs, a instaurer ce climat de rapports sociaux où l'affrontement devient possible, non-insécurisant et, partant, ouvre la voie à l'établissement de véritables consensus.

En se situant dans cette perspective et en donnant ainsi un encadrement conceptuel a son travail sur le terrain, l'auteur décrit d'une façon explicite et concrète les habiletés propres à l'agent de changement, les approches particulières visant à développer une organisation où règne une authentique collaboration. Ces approches déjà connues prennent comme cible respective le contexte socioéconomique, politique et culturel, les processus internes (leadership, communication) et le contenu c'est-à-dire tous les problèmes reliés aux stratégies, aux structures et au fonctionnement des organisations de travail.

En appuyant sur le contenu de l'intervention, l'auteur prend soin de reprendre le modèle original de la "recherche en action» (action research) en lui donnant beaucoup plus d'ampleur. De plus, il fournit de précieux conseils sur la nature des questions à poser, sur la manière de recueillir les données pertinentes, de les analyser et de les redonner aux individus et aux groupes qui ont contribué à les fournir. On reconnaîtra là la technique de l'enquête avec information en retour (survey-feedback).

Après avoir ainsi présenté les habiletés, les approches particulières, les techniques et également les phases d'une intervention, l'auteur consacre deux chapitres sur les méthodes d'amener des groupes à dégager des consensus, à développer et à maintenir des relations de collaboration. À ce sujet, il privilégie la thérapie de rôle (role therapy) qu'il décrit dans des termes relativement accessibles à ceux qui s'intéressent à ce domaine ou qui possèdent déjà quelques connaissances en sociologie et en psychologie sociale.

Afin d'illustrer de façon concrète son enseignement tiré surtout de son expérience, l'auteur utilise deux cas: une intervention qu'il a effectuée et qu'il relate succinctement pour appuyer son modèle conceptuel d'intervention et un deuxième cas où les habiletés nécessaires à l'intervention, les approches et les phases sont décrites. Par exemple, pour illustrer la phase initiale du contact avec le système-client et la rédaction d'un contrat d'intervention, l'auteur reproduit des échanges qu'il a enregistrés sur bande magnétique et en fait une analyse.

On peut reprocher à l'auteur l'utilisation de rubriques plutôt imprécises ou bien de termes «galvaudés» par l'usage qu'on en fait; par exemple, sa classification des organisations en collaborative, compétitive, passive et pyramidale alors qu'a la limite, on peut affirmer que toutes les organisations de travail sont de type pyramidal à cause de l'omniprésence de la hiérarchie. Il faut comprendre que le domaine de l'intervention dans les organisations de type américain, c'est-à-dire «développement organisationnel» est relativement nouveau et, par conséquent, il faut espérer qu'on trouvera les termes appropriés pour décrire ces expériences nouvelles. 
Tous ceux qui s'intéressent au changement organisationnel ou encore à l'établissement de consensus à l'échelle d'une organisation ou des groupes, la lecture d'un tel ouvrage sera profitable, puisqu'il se distingue de ceux déjà publiés à ce jour sur le sujet.

\section{Laurent BÉLANGER}

Université Laval

Le manager au quotidien Les dix rôles du cadre, par Henry Mintzberg, Montréal, Les éditions Agence d'Arc, 1984, 220 pp., ISBN 2-7081-0558-2 (français) et ISBN 0-13-610402-6 (américain)

Le manager au quotidien est la traduction de The Nature of Managerial Work paru pour la première fois chez Harper and Row en 1973. En 1980, Prentice Hall rééditait l'ouvrage et Le manager au quotidien est la traduction de cette édition qui ne comprend pas les annexes apparaissant dans l'édition originale. Cette attente de plus de dix ans illustre bien le problème de la disponibilité en français d'ouvrages classiques en management.

Le manager au quotidien est centré sur l'unique question: que font les cadres? Le livre est fondé sur les résultats d'observations et d'études empiriques dont les travaux effectués par l'auteur dans le cadre de sa thèse de doctorat. Le livre n'est toutefois pas la publication de la thèse. Il est agréable d lire et s'adresse à un large public, notamment aux managers eux-mêmes.

Dans le deuxième chapitre du livre consacré aux conceptions contemporaines sur le travail du cadre, l'auteur s'en prend à l'approche classique qui définit le travail du cadre à l'aide d'un ensemble de fonctions telles que la planification, l'organisation, le personnel, la direction, la coordination, le rapport et le budget. Selon l'auteur, lorsqu'on observe un cadre au travail, il est impossible de relier ses activités aux fonctions de POPDCORB. Ces fonctions ne décrivent pas le travail que fait le cadre, mais qualifient certains objectifs vagues de son travail. Selon Mintzberg, les écrits de l'école classique sont de peu d'utilité et ils ont pendant longtemps bloqué la recherche d'une compréhension plus profonde du travail du cadre. Se situant parmi l'école de l'activité du leader, l'auteur s'est intéressé à la fois aux caractéristiques et au contenu du travail du cadre. L'étude du contenu qui s'intéresse aux activités du cadre et au pourquoi de ces activités peut aboutir à des descriptions de fonctions ou de rôles.

Dans le troisième chapitre consacré aux caractéristiques du travail des cadres, l'auteur souligne le caractère peu structuré de ce travail. Le cadre se sent obligé de fournir une grande quantité de travail à un rythme soutenu. Ce travail est fragmenté et les interruptions sont fréquentes. Le cadre est attiré vers les éléments les plus actifs de son travail et il préfère les moyens oraux de communications, notamment dans le cadre de réunions programmées. Les subordonnées prennent entre le tiers et la moitié du temps que le cadre consacre aux contacts, tandis que les supérieurs n'en prennent que dix pour cent.

Le quatrième chapitre est consacré aux rôles professionnels du cadre. L'auteur y distingue dix rôles regroupés en trois rôles interpersonnels (symbole, leader, agent de liaison), trois rôles liés a l'information (observateur actif, diffuseur, porte-parole) et quatre rôles décisionnels (entrepreneur, régulateur, répartiteur de ressources, négociateur).

Dans le cinquième chapitre, l'auteur examine les variations existant entre postes d'encadrement. Ces variations peuvent être expliquées en faisant intervenir quatre ensembles de variables: des variables de l'environnement, des variables liées au poste, des variables liées à la personne et des variables relatives à la situation. Le niveau hiérarchique et la fonction supervisée paraissent avoir sur le travail du cadre plus d'influence qu'aucune autre variable.

Le sixième chapitre est consacré à l'impact de la science sur le travail du cadre. On y discute surtout de la contribution que les analystes pourraient apporter au travail de cadre. 